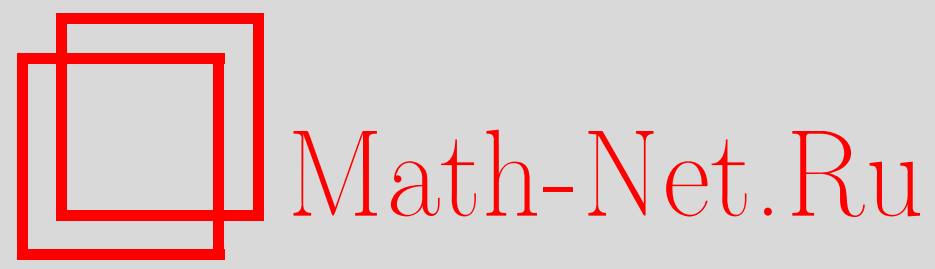

С. И. Адян, Проблема Бернсайда о периодических группах и смежные вопросы, Совр. пробл. матем., 2003, выпуск 1, 5-28

DOI: https://doi.org/10.4213/spm2

Использование Общероссийского математического портала MathNet.Ru подразумевает, что вы прочитали и согласны с пользовательским соглашением

http://www . mathnet.ru/rus/agreement

Параметры загрузки:

IP : 52.6 .47 .48

26 апреля 2023 г., 09:00:10 


\title{
Проблема Бернсайда о периодических группах и смежные вопросы
}

\author{
С.И. Адян
}

В работах П. С. Новикова и С. И. Адяна, опубликованных в $1959-$ 1975 гг., был создан новый метод исследования периодических групп, основанный на классификации периодических слов посредством сложной совместной индукции. Метод был создан для решения известной проблемы Бернсайда о периодических группах, но он позволил авторам решить и ряд других трудных проблем теории групп. В настоящей статье дается расширенный обзор как результатов, содержащихся в представленном цикле работ, так и других значительных результатов, полученных после 1975 года С. И. Адяном и другими авторами на базе созданной теории и ее модификаций.

\section{Введение}

Проблема Бернсайда о периодических группах фиксированного периода была поставлена известным английским ученым Бернсайдом в 1902 году в следующей форме (см. [1]).

Пусть $a_{1}, a_{2}, \ldots, a_{m}$ - конечное число независимых элементов, порождающих группу $G$, в которой для любого элемента $x$

Работа выполнена при финансовой поддержке Российского фонда фундаментальных исследований и Программы поддержки ведущих научных школ.

(C) Адян С.И., 2003 
выполнено соотношение $x^{n}=1$, где $n$ - данное целое число. Будет ли определенная таким образом группа конечной, и если да, то каков ее порядок?

Впоследствии группы, определенные $m$ порождающими и тождественным соотношением $x^{n}=1$, получили название свободны $x$ бернсайдовых групn периода $n$ и обозначение

$$
B(m, n)=\left\langle a_{1}, a_{2}, \ldots, a_{m} ; x^{n}=1\right\rangle .
$$

Более общий вопрос о локальной конечности периодических групп без ограничения на порядки элементов получил название "общая проблема Бернсайда".

В 1950 году в работе В. Магнуса [37] была сформулирована еще одна проблема, которая тесно связана с проблемой Бернсайда. Магнус назвал еe "restricted Burnside Problem", а в русской алгебраической литературе по инициативе И. Н. Санова [28] она была названа "ослабленная проблема Бернсайда". В ней требовалось выяснить, существует ли максимальная конечная периодическая группа с данным числом порождающих $m$ и фиксированным периодом $n$. Связь ослабленной проблемы с основной проблемой Бернсайда сводится к тому, что если бы не существовало бесконечных периодических групп, то группа $B(m, n)$ и была бы максимальной конечной периодической группой при этих $m$ и $n$. На самом деле оказалось, что при достаточно больших периодах $n$ ослабленная проблема имеет положительное решение, хотя свободные периодические группы $B(m, n)$ достаточно большого периода не только бесконечны, но и имеют бесконечное число различных факторгрупп (см. раздел 3$){ }^{1}$ Проблема Бернсайда привлекала внимание выдающихся алгебраистов многих стран в силу естественности и максимальной простоты своей постановки. Очевидно, любая конечная группа удовлетворяет тождественному соотношению $x^{n}=1$, где $n$ есть порядок этой группы. Естественно возникает вопрос о верности обратного утверждения. В результате многочисленных исследований был получен положительный

\footnotetext{
${ }^{1}$ Позже ослабленной проблемой Бернсайда занимались также И.Н. Санов, Г. Хигман, А.И. Кострикин, Е.И. Зельманов и др. Все они работали в направлении, намеченном Магнусом, т.е. исследовали лиевы кольца с тождеством Энгеля. Именно за результаты по ослабленной проблеме Бернсайда Ефиму Зельманову была присуждена Филдсовская премия.
} 
ответ на поставленный вопрос для некоторых специальных классов групп, а также возникли различные варианты этой проблемы для разных алгебраических систем. Однако сама проблема Бернсайда в ее первоначальной формулировке оставалась открытой в течение многих десятилетий.

В своей монографии по истории комбинаторной теории групп В. Магнус дает подробный анализ истории исследований по проблеме Бернсайда и при этом отмечает (см. [29, с. 57]): “ Само собой напрашивается сравнение влияния проблемы Бернсайда на комбинаторную теорию групп с влиянием последней теоремы Ферма на развитие алгебраической теории чисел".

Положительный ответ на вопрос Бернсайда для $n=3$ был получен самим Бернсайдом в [1] (1902), для $n=4-$ И. Н. Сановым в [27] (1940) и для $n=6$ - М. Холлом в [34] (1958). Кроме того, была доказана локальная конечность всех матричных групп при ограниченном периоде элементов самим Бернсайдом в [2] (1905), а при неограниченных периодах - И. Шуром в [37] (1911).

В 1964 году Е. С. Голод впервые доказал, что общая проблема Бернсайда имеет отрицательное решение, т.е. существуют бесконечные 2-порожденные периодические группы с неограниченными периодами элементов [20]. Позже такого рода примеры были построены также С. В. Алешиным [18], Р. И. Григорчуком [21] и другими авторами.

Отрицательное решение проблемы Бернсайда было получено в фундаментальной работе [4], опубликованной в 1968 году в серии совместных статей двух авторов в журнале Известия $A H$ СССР. Сер. матем. Т. 32, №oo 1-3. Была доказана следующая теорема.

ТЕОРемА 1. Для любого нечетного периода $n \geqslant 4381$ и любого числа порождающих $m \geqslant 2$ свободная периодическая группа $B(m, n)$ бесконечна.

Для доказательства этой теоремы авторами была создана новая теория, суть которой заключается в классификации периодических слов данного нечетного периода в групповом алфавите, а также преобразований таких слов на базе периодических 
соотношений. Характерной особенностью теории является доказательство большого числа утверждений совместной индукцией по натуральному параметру, причем определения используемых понятий вводятся попутно в ходе той же индукции.

Здесь уместно напомнить о том, что еще в 1959 году П. С. Новиков в своей заметке [3] анонсировал отрицательное решение проблемы Бернсайда в более сильной форме. Однако при реализации намеченного им плана встретились серьезные трудности. В 1960 году П. С. Новиков предложил автору настоящего обзора работать вместе по осуществлению намеченного им замысла. В результате нашей 8-летней совместной работы и была создана новая теория, позволившая дать отрицательное решение проблемы Бернсайда в основном ее варианте для нечетных периодов $n \geqslant 4381$. Хотя П. С. Новиков публиковал заметку [3] под давлением сложившихся обстоятельств, автор настоящего обзора убежден, что факт публикации анонса в [3] сыграл положительную роль в активизации работы авторов над проблемой. Без этого анонса могло бы не быть того энтузиазма, с которым мы работали эти годы, а возможно, не было бы и самого результата. Во всяком случае, до этого алгебраисты думали лишь о положительном решении проблемы Бернсайда, а некоторые даже говорили о "предложении Бернсайда", а не о проблеме. Так один из тогдашних лидеров советской алгебры профессор А.Г. Курош в своей известной монографии “Теория групп”, вышедшей в 1953 году, писал, что решение вопроса о локальной конечности периодических групп "не удается получить даже при условии, что порядки элементов ограничены в совокупности". Ясно, что здесь подразумевается положительное решение этого вопроса. Не является случайным и тот факт, что первые примеры локально бесконечных периодических групп были построены Е. С. Голодом после публикации заметки [3].

Характерной особенностью созданной в [4] теории является то, что почти все утверждения этой теории, включая определения основных понятий, вводятся и доказываются одной совместной индукцией по натуральному параметру, который называется рангом. Это, конечно, создает определенные трудности при чтении работы, так как читатель должен некоторое время иметь дело 
с понятиями и утверждениями, которые будут определены или доказаны ниже.

В 1975 году была опубликована монография автора [10], в которой рассматриваемая теория была усовершенствована и все полученные к этому времени результаты, в том числе и теорема 1 , были распространены на нечетные периоды $n \geqslant 665$. По сравнению с первоначальным текстом совместных публикаций 1968 года в монографии [10] изложение было существенно упрощено. Кроме того, книга снабжена указателем, позволяющим читателю легко находить нужные определения, к которым он вынужден периодически обращаться при чтении доказательств.

\section{1. Краткое описание теории}

Основные идеи созданной двумя авторами теории здесь будут изложены в версии, которая была опубликована в монографии [10]. Для этого фиксируем нечетный период $n \geqslant 665$ и два числовых параметра $p=9$ и $q=90$. Будем рассматривать слова в групповом алфавите

$$
a_{1}, a_{2}, \ldots, a_{m}, a_{1}^{-1}, a_{2}^{-1}, \ldots, a_{m}^{-1}, \quad m>1
$$

Слово $A$ в алфавите (1) называется минималънъм периодом ранга 1 , если $A$ есть кратчайший период несократимого слова $A^{n}$. Период $A$ называется элементарным периодом ранга 1 , если в слово $A^{n}$ не входит никакое подслово вида $B^{t} B_{1}$, где $B_{1}$ есть начало периода $B$, длина $|B|$ меньше $|A|$ и $|E| \geqslant p|B|$. Если же в слово $A^{n}$ входит подслово такого вида, то $A$ называется периодом ранга 2 , а $A^{n}$ - периодическим словом ранга 2 с периодом $A$.

Для точного определения индукцией по натуральному параметру $\alpha$ понятий периода ранга $\alpha$ и элементарного периода ранга $\alpha$ требуется попутно ввести определения ряда других понятий, связанных с рангом $\alpha$, как-то:

1. ядро ранга а данного слова;

2. приведенное слово ранга $\alpha$; 
3. поворот ранга $\alpha$;

4. отношение эквивалентности слов в ранге $\alpha$;

5. взаимная нормированность двух вхождений в ранге $\alpha$;

6. операчия смыкания ранга а для данных двух слов и т. д.

Точные определения всех этих понятий можно найти в [10, гл. $1, \S 4]$. Здесь же мы ограничимся лишь указанием некоторых существенных особенностей рассматриваемой системы понятий.

Допустим, что для ранга $\alpha-1 \geqslant 0$ уже определены следующие понятия:

1. приведенные слова ранга $\alpha-1$ (множество всех таких слов обозначается через $\mathcal{R}_{\alpha-1}$, причем $\mathcal{R}_{0}$ есть множество всех несократимых слов в алфавите $(1)$ и $\mathcal{R}_{i} \subset \mathcal{R}_{i-1}$ при всех $i \geqslant 1)$

2. ядра ранга $\alpha$ данного слова $X \in \mathcal{R}_{\alpha-1}$ (ядрами ранга 0 слова $X$ из $\mathcal{R}_{0}$ являются все вхождения букв в слово $X$, а в общем случае это вхождения некоторых подслов в $X)$;

3. отношение эквивалентности в ранге $\alpha-1$ для слов $X, Y$ из $\mathcal{R}_{\alpha-1}$ (обозначается через $X \stackrel{\alpha-1}{\sim} Y$, причем $X \stackrel{0}{\sim} Y$ означает, что $X$ и $Y$ равны графически);

4. элементарный период ранга $\alpha-1$ при $\alpha>1$ (точное определение элементарных периодов ранга 1 дано выше).

Слово $A$ в алфавите (1) называется периодом ранга $\alpha$, если слово $A^{n}$ принадлежит множеству $\mathcal{R}_{\alpha-1}$ и имеет такое ядро ранга $\alpha-1$, длина которого меньше двух длин периода $A$. При этом слово $A^{n}$ называется периодическим словом ранга $\alpha$. Оно будет содержать много ядер ранга $\alpha-1$, которые равномерно распределены во всех периодах $A$. Среди периодов ранга $\alpha$ мы сначала выбираем так называемые минимальные периоды ранга $\alpha$. Для этого мы рассматриваем всевозможные слова $Y$, эквивалентные $A^{n}$ в ранге $\alpha-1$. Мы говорим, что период $A$ не минимален в ранге $\alpha$, если среди слов, эквивалентных $A^{n}$ в ранге $\alpha-1$, найдется такое слово вида $P B^{t} Q$, где $t \geqslant p, B$ есть период ранга $\alpha$, в слове $B^{n}$ на каждый период $B$ приходится меньше ядер ранга $\alpha-1$, чем в слове $A^{n}$ приходится на период $A$; и в то же время подслово $B^{t}$ содержит не меньше ядер ранга $\alpha-1$, чем их приходится 
на 3 периода $A$ слова $A^{n}$. В противном случае мы называем $A$ минимальным периодом ранга $\alpha$.

Среди минимальных периодов ранга $\alpha$ выбираются элементарные периоды ранга $\alpha$. При этом каждый минимальный период ранга $\alpha$, который не является элементарным периодом ранга $\alpha$, оказывается периодом следующего ранга $\alpha+1$.

Элементарными словами ранга $\alpha$ с периодом $A$ называются внутренние сегменты слов, эквивалентных в ранге $\alpha-1$ слову $A^{n}$ с элементарным периодом $A$. Они порождаются так называемыми порождающими вхождениями. Элементарное слово $E$ ранга $\alpha$ называется элементарной $t$-степенъю ранга $\alpha$, если в его порождающем вхождении содержится больше ядер ранга $\alpha-1$, чем их приходится на $t-1$ периодов слова $A^{n}$.

Далее вводится понятие нормированного вхождения элементарного слова $E$ ранга $\alpha$ в данное слово $X$ из множества $\mathcal{R}_{\alpha-1}$. Такие вхождения обладают многими свойствами их порождающих вхождений.

На основе ряда установленных свойств элементарных слов ранга $\alpha$ и их вхождений вводится понятие $r$-nоворота ранга $\alpha$. Пусть $r \geqslant 9$ и $A=A_{1} A_{2}$ есть элементарный период ранга $\alpha$. Переход

$$
X \stackrel{0}{\sim} T A^{t} A_{1} Q \rightarrow T A^{-n+t+1} A_{2}^{-1} Q \stackrel{0}{\sim} Y
$$

называется простым $r$-поворотом ранга $\alpha$ данного вхождения $T * A^{t} A_{1} * Q$ элементарного слова $A^{t} A_{1}$, если слова $X, Y$ лежат в $\mathcal{R}_{\alpha-1}$ и в обоих вхождениях $T * A^{t} A_{1} * Q$ и $T * A^{-n+t+1} A_{2}^{-1} * Q$ содержатся нормированные вхождения элементарных $r$-степеней ранга $\alpha$ с периодами $A$ и $A^{-1}$ соответственно. Очевидно, переход (2) равносилен применению определяющего соотношения $1=A^{-n}$ с последующим сокращением в свободной группе.

Понятие $r$-поворота ранга $\alpha$ естественным образом распространяется на переходы вида $X_{1} \rightarrow Y_{1}$, где слова $X_{1}$ и $Y_{1}$ эквивалентны в ранге $\alpha-1$ словам $X$ и $Y$ соответственно. Если $r \geqslant k \geqslant 9$, то всякий $r$-поворот ранга $\alpha$ есть также $k$-поворот того же вхождения.

Некоторые $q$-повороты ранга $\alpha$ называются реальными поворотами ранга $\alpha$. Два слова $X, Y$ из множества $\mathcal{R}_{\alpha-1}$ называются 
эквивалентными в ранге $\alpha$ (пишем $X \stackrel{\alpha}{\sim} Y$ ), если либо $X \stackrel{\alpha-1}{\sim} Y$, либо можно указать некоторую последовательность реальных поворотов ранга $\alpha$, переводящую $X$ в $Y$.

При рассмотрении последовательностей реальных поворотов ранга $\alpha$ важную роль играет понятие совпадения индивидуальностей вхождений элементарных $p$-степеней ранга $\alpha$ в слова рассматриваемой последовательности. Для простого поворота ранга $\alpha$ это понятие вводится следующим образом. Выделенные в повороте (2) вхождения периодических слов по определению соответствуют друг другу по индивидуальности в (2), а каждое содержащееся в $T$ (или в $Q$ ) нормированное вхождение элементарной $p$-степени $E$ ранга $\alpha$ в слово $X$ естественным образом соответствует по индивидуальности в (2) лежащему точно в том же положении в $T$ (или в $Q$ ) вхождению слова $E$ в слово $T A^{-n+t+1} A_{2}^{-1} Q$ при условии, что оно также нормированное. Это отношение транзитивно распространяется на любые последовательности реальных поворотов ранга $\alpha$, т.е. на отношение $X \stackrel{\alpha}{\sim} Y$

Далее для нормированных вхождений элементарных $p$-степе-

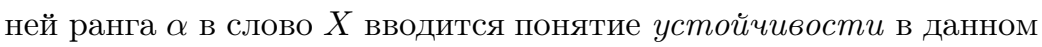
реальном повороте $X \rightarrow Y$ ранга $\alpha$. Нормированное вхождение $V$ элементарной $p$-степени $E$ ранга $\alpha$ в слово $X$ называется ядром ранга $\alpha$ слова $X$, если оно устойчиво (т.е. не затрагивается) в любом реальном повороте ранга $\alpha$, не являющемся поворотом самого вхождения $V$, и никакое его нормированное продолжение не обладает этим свойством. Если два слова эквивалентны в ранге $\alpha$, то они содержат одинаковое число ядер ранга $\alpha$. Таким образом, число ядер ранга $\alpha$ данного слова является инвариантом эквивалентных преобразований в ранге $\alpha$.

Приведенное слово ранга $\alpha-1$ называется приведенным словом ранга $\alpha$ (пишем $X \in \mathcal{R}_{\alpha}$ ), если все его ядра ранга $\alpha$ содержат не более чем $n-176$ периодов.

На множестве $\mathcal{R}_{\alpha-1}$ следующим образом определяется операция умножения слов, называемая смыканием ранга $\alpha$ :

$$
[X, Y]_{\alpha}=P Q \quad \Longleftrightarrow \quad\left(X \stackrel{\alpha}{\sim} P T \quad \& \quad Y \stackrel{\alpha}{\sim} T^{-1} Q \quad \& \quad P Q \in \mathcal{R}_{\alpha-1}\right)
$$


Доказывается, что эта операция на множестве $\mathcal{R}_{\alpha-1}$ определена однозначно с точностью до эквивалентности в ранге $\alpha$ и ассоциативна. Более того, множество классов эквивалентности в ранге $\alpha$ слов из $\mathcal{R}_{\alpha-1}$ с этой бинарной операцией образует группу, изоморфную группе

$$
B(m, n, \alpha)=\left\langle a_{1}, a_{2}, \ldots, a_{m} ;\left\{A^{n}=1 \mid A \in \bigcup_{i=1}^{\alpha} \mathcal{A}_{i}\right\}\right\rangle
$$

где $\mathcal{A}_{i}$ есть множество всех элементарных периодов ранга $i$. В частности, для любых слов $X, Y$ из $\mathcal{R}_{\alpha-1}$ выполнено соотношение

$$
X \stackrel{\alpha}{\sim} Y \quad \Longleftrightarrow \quad X=Y \text { в группе } B(m, n, \alpha) .
$$

В пределе мы получаем группу, изоморфную свободной бернсайдовой группе $B(m, n)$. Тем самым завершается доказательство следующей теоремы.

ТЕОРЕма 2. При нечетных $n \geqslant 665$ свободная бернсайдова группа $B(m, n)$ изоморфна группе

$$
B(m, n, \infty)=\left\langle a_{1}, a_{2}, \ldots, a_{m} ;\left\{A^{n}=1 \mid A \in \bigcup_{i=1}^{\infty} \mathcal{E}_{i}\right\}\right\rangle .
$$

Таким образом, изложенный в работах [2] и [8] подход заключается в том, что, начиная со свободной группы $B(m, n, 0)=F_{m}$, мы последовательно добавляем определяющие соотношения вида $A^{n}=1$, сначала для всех элементарных периодов ранга 1 , затем для всех элементарных периодов ранга 2 и т. д. При этом само понятие элементарного периода ранга $\alpha$, так же как и все сопутствующие ему понятия для ранга $\alpha$, определяются на базе ранее построенного отношения " $\alpha-1$ ", т.е. на базе отношения равенства слов в уже построенной группе $B(m, n, \alpha-1)$.

Группы $B(m, n)$ могут быть заданы независимой системой определяющих соотношений. Такая система определяющих соотношений для группы $B(m, n)$ получается путем выбора в каждом классе взаимно сопряженных в группе $B(m, n, \alpha-1)$ элементарных периодов ранга $\alpha$ по одному элементарному периоду с дополнительным условием, чтобы они не были сопряжены также 
и отрицательным степеням уже выбранных элементарных периодов данного ранга. Доказывается, что выбранные таким образом множества элементарных периодов ранга $\alpha$ независимы. Обозначим их через $\overline{\mathcal{E}}_{\alpha}$. В результате получается следующее усиление теоремы 2.

Теорема 3. При нечетных $n \geqslant 665$ свободная бернсайдова групnа $B(m, n)$ имеет следующее экономное задание:

$$
B(m, n)=\left\langle a_{1}, a_{2}, \ldots, a_{m} ;\left\{A^{n}=1 \mid A \in \bigcup_{i=1}^{\infty} \overline{\mathcal{E}}_{i}\right\}\right\rangle,
$$

где ни одно из определяющих соотношений нельзя выкинутъ.

Доказательство бесконечности группы $B(m, n)$ опирается на существование бесконечных двубуквенных слов, которые не содержат подслов вида $A^{3}$ (так называемых последовательностей Туэ-Аршона). Роль такой последовательности здесь может играть последовательность слов $C_{i}$ в двубуквенном групповом алфавите (1), которая определяется индуктивно на основе следующих равенств:

$$
C_{1}=a_{1}, \quad C_{i+1}=C_{i} a_{2} C_{i}^{-1} .
$$

Несложный анализ показывает, что все эти слова $C_{i}$ не содержат квадратов слов.

Среди многочисленных утверждений, доказываемых совместной индукцией, в нашей теории есть следующая простая, но ключевая лемма.

Лемма 1. Если слово $X$ не содержит подслов вида $A^{9}$, то оно приведено в любом ранге $\alpha$ и не эквивалентно никакому отличному от него несократимому слову.

Легко доказывается, что каждое слово в алфавите группы $B(m, n)$ равно в ней некоторому слову, которое является приведенным во всех рангах. Очевидно, если два слова эквивалентны в некотором ранге $\alpha$, то они равны в группе $B(m, n)$. Доказывается, что для слов, являющихся приведенными во всех рангах, верно и обратное, т.е. верна 
Лемма 2. Если слова $X$ и $Y$ являются приведенными во всех рангах, то

$$
X=Y \text { в } B(m, n) \quad \Longleftrightarrow \quad X \stackrel{\alpha}{\sim} Y \text { при некотором } \alpha
$$

В силу лемм 1 и 2 слова $C^{i}$ задают бесконечное число попарно неравных друг другу элементов группы $B(m, n)$. Тем самым завершается доказательство следующего аналога теоремы 1.

ТЕОРЕмА 4. При $m \geqslant 1$ и нечетных $n \geqslant 665$ свободная бернсайдова группа $B(m, n)$ бесконечна.

Так как свободная бернсайдова группа $B(m, n)$ является факторгруппой групп $B(m, n k)$ при любых $k>1$, то из бесконечности группы $B(m, n)$ непосредственно следует также бесконечность бернсайдовых групп $B(m, n k)$ при нечетных $n \geqslant 665$ и любых $k>1$.

Заметим, что до сих пор $n=665$ остается наименъшим значением периода, при котором удалось доказать бесконечность свободной бернсайдовой группъ $B(m, n)$.

\section{2. Свойства свободных периодических групп нечетного периода}

Как это часто бывает в математике, при появлении нового метода, решающего проблему, не поддававшуюся долгое время усилиям математиков, созданный авторами метод исследования периодических групп вскоре нашел ряд других важных приложений. В том же 1968 году вышли еще две совместные статьи [5] и [6], в которых было доказано, что рассматриваемые группы $B(m, n)$ не могут быть заданы с помощью конечного числа определяющих соотношений и в них разрешимы проблемы равенства слов и сопряженности. Было также доказано, что все абелевы подгруппы этих групп конечны. В монографии [10] все результаты совместных статей двух авторов [4]-[6] были доказаны уже для любых нечетных периодов $n \geqslant 665$. Это существенное понижение границы для $n$ в монографии было достигнуто в результате дальнейшего усовершенствования метода. 
Разрешимость проблемы распознавания равенства слов для $B(m, n)$ вытекает из принципа эффективности (см. [10, I.5.4]), согласно которому все рассматриваемые множества слов, функции и отношения между словами и их подсловами алгоритмически эффективны, что также проверяется попутно совместной индукцией. Для доказательства разрешимости проблемы сопряженности кроме принципа эффективности используется следующая лемма.

ЛЕмма 3. $B$ группе $B(m, n)$ всякое непустое слово сопряжено некоторой степени $A^{r}$ некоторого элементарного периода $A$ некоторого ранга $\alpha$, причем этот элементарный период, его степень $r$ и ранг $\alpha$ можно найти алгоритмически.

По этой лемме вопрос о распознавании сопряженности любых двух слов сводится к распознаванию сопряженности соответствующих элементарных периодов, что, в свою очередь, легко проверяется на основе принципа эффективности.

С помощью последовательности Туэ-Аршона строятся примеры элементарных периодов сколь угодно большого ранга, откуда следует, что рассматриваемые группы $B(m, n)$ не могут быть заданы с помощью конечного числа определяющих соотношений, так как соотношение $A^{n}=1$ для периода $A$ данного ранга не может быть выведено из таких же соотношений для периодов меньших рангов.

Доказательство утверждения, что при нечетных $n \geqslant 665$ всякая коммутативная подгруппа группы $B(m, n)$ циклическая и ее порядок есть делитель числа $n$, получено на основе леммы:

ЛЕмма 4. Любой неединичный элемент $x$ группы $B(m, n)$ равен в $B(m, n)$ некоторой степени некоторого элемента $z$, имеющего порядок $n$, причем все коммутирующие с $x$ элементы являются степенями того же элемента z.

Из этой леммы легко вытекает тривиальность центра группы $B(m, n)$.

В работе [9] доказано, что все конечные подгруппы в $B(m, n)$ также являются циклическими. Там же установлено, что группа 
$B(2, n)$ содержит подгруппу, изоморфную $B(3, n)$. Несколько позже в кандидатской диссертации В. Л. Ширваняна (1976) было доказано, что наш метод позволяет доказать вложимость $B(\infty, n)$ с бесконечным числом порождающих в группу $B(2, n)$. Отсюда легко следует

Теорема 5. При $m \geqslant 1$ и нечетных $n \geqslant 665$ свободная бернсайдова группа $B(m, n)$ содержит бесконечные убывающие и бесконечные возрастающие цепочки вложенных подгрупп.

На самом деле, в группах $B(m, n)$ существуют также бесконечные убывающие и бесконечные возрастающие цепочки нормальных подгрупп. При составных $n=r s$, где $r \geqslant 665$ нечетно и $s>1$, это доказывается несложно. Несколько сложнее доказательство аналогичного свойства при любых простых $n \geqslant 665$ и $m>65$. Для доказательства последнего утверждения в работе [13] была рассмотрена новая модификация первоначальной теории. В ней наряду с поворотами периодических слов используются также повороты так называемых метапериодических слов, которые не имеют периодичности. Хотя такие повороты появляются только в ранге 1 , они обеспечивают возможность бесконечного числа последовательных нетривиальных факторизаций группы $B(m, n)$, в том числе и при простых $n \geqslant 665$.

Эта новая конструкция позволила нам впервые построить примеры конечно порожденных групп, удовлетворяющих тождеству $x^{n}=1$ и имеющих неразрешимую проблему равенства.

Многие из перечисленных выше свойств групп $B(m, n)$ можно рассматривать как существенные усиления утверждения о бесконечности групп $B(m, n)$. Они аналогичны известным свойствам неабелевых свободных групп. В монографии [10] был доказан еще более неожиданный результат о том, что рассматриваемые группы $B(m, n)$ имеют экспоненциальный рост. Функция роста $\gamma(s)$ конечно порожденной группы определяется как число различных элементов, которые представимы в виде произведения не более чем $s$ порождающих. В частности, было установлено, что в группе $B(2, n)$ (при нечетных $n \geqslant 665$ ) имеется не менее $4 \cdot(2.9)^{s-1}$ различных элементов, которые представимы в виде слова длины $s$. Заметим, что это очень близко к свободной группе, так как для свободной группы с двумя порождающими число различных элементов данной длины $s$ в точности равно $4 \cdot 3^{s-1}$. Заметим, что 
для решения самой проблемы Бернсайда, по существу, требовалось лишь ответить на вопрос: может ли функция роста группы $B(m, n)$ быть неограниченной?

В 1982 году в работе [15] была доказана следующая теорема.

ТЕОРемА 6. При $m \geqslant 1$ и нечетных $n \geqslant 665$ свободная бернсайдова группа $B(m, n)$ не является аменабельной и симметричное случайное блуждание на $B(m, n)$ не является возвратным.

Тем самым впервые были найдены неаменабельные группы, удовлетворяющие нетривиальному тождеству.

Результат о случайных блужданиях, в свою очередь, явился отрицательным ответом на вопрос, поставленный еще в 1959 году известным американским вероятностником Кестеном [36].

Оба утверждения теоремы 6 были высказаны автором в виде гипотез еще в 1977 году в работе [14, с. 10]. Доказательство этих результатов в [15] опирается на результат Р. И. Григорчука (см. [22]), который свел вопрос об оценке спектрального радиуса симметрического случайного блуждания на группе $G$ с $m$ порождающими к оценке показателя роста нормальной подгруппы свободной группы $F^{m}$, факторизация по которой дает группу G. В 1977 году такое сведение еще не было известно. Для оценки спектрального радиуса случайного блуждания на группе $B(m, n)$ была указана такая система определяющих соотношений этой группы, которая удовлетворяет условию Дэна, причем скорость сходимости соответствующего алгоритма Дэна не меньше 228, а относительная скорость сходимости не меньше $1 / 3$. Напомним, что условием Дэна для данной группы $G$, заданной множеством определяющих соотношений $A$, называется требование, чтобы каждое равное 1 в $G$ слово имело вид $P E Q$, где $E$ есть кусок левой части $A$ одного из определяющих соотношений, длина которого больше половины длины $A$. При этом скоростью сходимости алгоритма Дэна относительно $A$ называется максимальное число $\delta_{R}$ такое, что всегда разность длин $|E|-|Q P|$ не меньше $\delta_{R}$, а относительной скоростъю сходимоcmu для $R$ - максимальное число $\gamma_{R}$, удовлетворяющее условию $|E|-|Q P| \geqslant \gamma_{R} \cdot|P E Q|$. 
Как было отмечено в [31], соединение содержащихся в работах [30] и [15] конструкций позволяет легко получить следующую интересную теорему.

ТЕОРемА 7. При $m \geqslant 1$ и нечетных $n \geqslant 665$ свободная бернсайдова группа $B(m, n)$ может быть представлена как предел бесконечной последовательности групп, задаваемых конечным числом определяющих соотношений с условием Дэна,

$$
G_{0}, G_{1}, \ldots, G_{i}, G_{i+1}, \ldots
$$

где $G_{0}$ есть свободная группа с $m$ порождающими и каждая группа $G_{i+1}$ получается добавлением $\kappa$ заданию группъ $G_{i}$ одного соотношения вида $A^{n}=1$.

Напомним, что группы, удовлетворяющие условию Дэна, недавно с легкой руки Г. Громова почему-то были названы гunepболическими по Громову.

\section{3. Независимые системы групповых тождеств}

Уже в 1969 году автору стало ясно, что, модифицируя созданный нами метод, можно использовать его для построения групп с различными наперед заданными свойствами. Эта идея была впервые применена для решения известной проблемы конечного базиса теории групп. Требовалось выяснить, можно ли любую систему групповых тождеств привести к эквивалентной ей конечной системе тождеств, иначе говоря, можно ли любое многообразие групп задать конечной системой тождеств? Отрицательный ответ был получен в самой сильной форме. Была доказана

ТЕОрема 8. Cистема групповъх тождеств

$$
\left\{\left(x^{p n} y^{p n} x^{-p n} y^{-p n}\right)^{n}=1\right\}
$$

где $n \geqslant 1003$ - биксированное нечетное число, а $p$ - параметр, пробегающий все простые числа, является независимой, т.е. ни одно из этих тождеств не вытекает из остальных. 
Для доказательства этой теоремы требовалось по любому значению параметра $p=l$ построить некоторую группу, удовлетворяющую всем тождествам (5), кроме того из них, которое получается при $p=l$. Искомая группа $G_{l}$ строилась на основе некоторой модификации созданного нами метода. В этой модификации наряду со всеми понятиями работы [4] индукцией по рангу $\alpha$ определялось понятие допустимого периода ранга $\alpha+1$. Именно, минимальный период $D$ ранга $\alpha+1$ называется допустимым в ранге $\alpha$, если можно указать такие слова $A$ и $B$, что после подстановки их в левую часть тождества (5) при $p \neq l$ получается слово, которое сопряжено в группе $B(m, n, \alpha)$ некоторой степени периода $D$. В новой теории рассматриваются только такие повороты (2) ранга $\alpha$, в которых $A$ есть допустимый элементарный период ранга $\alpha$. Вносится соответствующая корректировка всех определений основных понятий. В частности, все ограничения на число периодов в ядрах ранга $\alpha$ приведенных слов ранга относятся только к тем ядрам, которые связаны с допустимыми периодами ранга $\alpha$. Искомая группа $G_{l}$ строится в новой теории аналогично тому, как в первоначальной теории строилась группа $B(m, n, \infty)$.

Истинность тождеств (5) при $p \neq l$ в построенной группе доказывается аналогично тому, как в первоначальной теории доказывалась истинность тождества $x^{n}=1$. При этом ключевую роль играет

Лемма 5. Если элементарный период D допустим в ранге $\alpha$ u в слово $D^{9}$ не входит никакая допустимая 17-степень ранга $\alpha$, то период D допустим и в некотором ранге $\alpha$.

Эта лемма существенно используется также в доказательстве того, что тождество (5) при $p=l$ в группе $G_{l}$ не выполняется.

\section{4. Некоммутативные аналоги аддитивной группы рациональных чисел}

Известно, что каждая коммутативная группа со свойством бесконечности пересечения любой пары ее циклических подгрупп изоморфна некоторой подгруппе аддитивной группы рациональных чисел. Поэтому это свойство можно считать характеристиче- 
ским свойством аддитивной группы рациональных чисел. В теории групп давно стоял открытый вопрос о существовании некоммутативных групп с таким же свойством, которые можно было называть некоммутативными аналогами аддитивной группы рациональных чисел. Очевидно, такая группа не должна иметь элементов конечного порядка. Тем не менее в 1971 году в работе [8] с помощью некоторой модификации нашей теории было впервые получено положительное решение этого вопроса. Построенные нами конечно порожденные некоммутативные группы без кручения $A(m, n)$, в которых пересечение любых двух нетривиальных подгрупп бесконечно, являются центральными расширениями групп $B(m, n)$ с циклическим центром. Они задаются в следующем виде:

$$
\begin{aligned}
& A(m, n)=\left\langle a_{1}, a_{2}, \ldots, a_{m}, d\right. \\
&\left.a_{j} d=d a_{j}, 1 \leqslant j \leqslant n, \quad\left\{A^{n}=d \mid A \in \bigcup_{i=1}^{\infty} \overline{\mathcal{E}}_{i}\right\}\right\rangle
\end{aligned}
$$

где $\overline{\mathcal{E}}_{i}$ - независимые множества элементарных периодов ранга $i$, которые использовались в экономном задании (4) для группы $B(m, n)$. Тем самым было установлено, что в нашей теории можно использовать не только периодические соотношения; достаточно, чтобы они содержали длинные периодические отрезки. В то время как подгруппы группы рациональных чисел либо являются циклическими, либо имеют бесконечное число порождающих, их некоммутативные аналоги $A(m, n)$ могут иметь любое конечное число $m \geqslant 2$ порождающих.

Основное свойство групп $A(m, n)$ заключается в следующем утверждении.

ЛЕмма 6. Любой элемент групп $A(m, n)$ равен некоторой степени элемента $d$, порождающего центр группь, причем $d$ имеет бесконечный порядок.

Обозначим через $\bar{A}(m, n)$ результат добавления к соотношениям группы $A(m, n)$ еще одного соотношения $d^{n}=1$. Из леммы 6 легко следует

Теорема 9. Счетная группа $\bar{A}(m, n)$ допускает только дискретную топологию. 
В самом деле, из леммы 6 следует, что в группе $\bar{A}(m, n)$ любой неединичный элемент является решением одного из конечного числа уравнений

$$
x^{n}=d, \quad x^{n}=d^{2}, \quad \ldots, \quad x^{n}=d^{n-1} .
$$

Если оснастить группу $\bar{A}(m, n)$ какой-то топологией, то в полученной топологической группе множество всех решений каждого уравнения из конечного множества (7), а значит, и их объединение будет замкнутым множеством. Следовательно, дополнение к этому замкнутому множеству будет открытым и будет содержать единственный элемент - единицу группы.

Вопрос о существовании счетных групп с таким свойством был поставлен А. А. Марковым и оставался открытым несколько десятилетий.

\section{5. Периодические произведения групп}

В работе [11] было установлено, что с использованием некоторой модификации нашей теории могут быть построены новые операции умножения групп, названные периодическими произведениями данного периода $n$. Эта операция умножения обладает всеми свойствами классических операций свободного и прямого произведений групп, в том числе и свойством наследственности по подгруппам. Последнее свойство означает, что если в периодическом произведении $H$ двух или более групп в каждой компоненте выбрать по подгруппе, то эти подгруппы порождают в $H$ периодическое произведение самих подгрупп.

В работе [12] был установлен следующий критерий простоты группы, являющейся периодическим произведением нечетного периода данного семейства групп.

ТеОрема 10. Периодическое произведение нечетного периода $n \geqslant 665$ данного семейства групп является простой группой в том и только том случае, когда каждая компонента этого произведения становится единичной группой при добавлении тождества $x^{n}=1$. 
Этот критерий простоты позволяет строить новые серии конечно порожденных бесконечных простых групп в многообразиях периодических групп нечетного составного периода $n k$, где $k>1$ и $n \geqslant 665$. В частности, доказано, что для любого множества нечетных простых чисел $M$, содержащего хотя бы одно число $p \geqslant 664$, можно построить счетную периодическую группу $G$ со спектром $M$. Это означает, что множество всех порядков элементов группы $G$ совпадает с $M$.

\section{6. Некоторые результаты других авторов}

В 1973 году известный английский математик Джон Бриттон предпринял попытку дать альтернативное отрицательное решение проблемы Бернсайда. В своей работе [32] он претендовал на независимое решение проблемы Бернсайда. При подготовке к печати монографии [10] перед автором встал вопрос, как ссылаться на эту работу. Настораживал тот факт, что в 282-страничной статье Бриттона доказывалось существование нечетного периода $n$, при котором группа бесконечна, но не были указаны конкретные значения для периода группы. Поэтому мы тщательно проверили работу [32]. Эта проверка показала, что доказательство Бриттона основано на противоречивой системе неравенств между используемыми им параметрами. Это противоречие было отмечено в предисловии к монографии [10]. Позже в письме [33] Бриттон и сам признал со ссылкой на [10], что его доказательство было ошибочным.

Через несколько лет после издания монографии [10] у авторов появились последователи, которые не только разобрались в новом методе, но и смогли применить его для получения новых результатов. Так, А. Ю. Ольшанский рассмотрел геометрическую модификацию метода Новикова-Адяна с использованием диаграмм Ван Кампена, которые являются известным эквивалентом выводов равенств слов из данной системы определяющих соотношений. Многочисленные результаты, полученные на этом пути им и его учениками, приведены в книге [26]. Наиболее ярким из 
них следует признать его результат 1982 года о существовании для простых $n>10^{75}$ бесконечной 2-порожденной группы, порядки всех собственных подгрупп которой являются делителями числа $n$ (так называемые "монстры Тарского"). В работе [19] В.С. Атабекян и С.В. Иванов распространили этот результат на любые нечетные периоды $n>10^{80}$. В 1989 году во время обсуждения кандидатской диссертации ученика А. Ю. Ольшанского В. С. Губы ученики А. Ю. Ольшанского высказали предположение, что предложенный А. Ю. Ольшанским метод существенно отличается от оригинальной теории Новикова-Адяна, так как он позволяет строить такие монстры Тарского, которые не были построены с помощью первоначальной теории. Для опровержения этой иллюзии мы с И.Г. Лысенком обещали и в 1991 году опубликовали совместную работу [17], в которой была доказана следующая теорема.

ТЕОРема 11. Для любого нечетного периода $n \geqslant 1003$ существует 2-порожденная бесконечная группа, всякая собственная подгруппа которой содержится в некоторой ииклической подгруппе порядка $n$.

Доказательство этой теоремы было проведено на оригинальном языке монографии [10] и основано на изложенной в последней главе этой книги технике при тех же значениях периода $n \geqslant 1003$. После этого не осталось никаких сомнений в том, что используемый А. Ю. Ольшанским подход есть несколько упрощенная и ослабленная модификация метода Новикова-Адяна с использованием геометрического языка диаграмм Ван Кампена для данной системы определяющих соотношений вместо цепочки выводов из этих соотношений. Разумеется, это не умаляет значение результатов, полученных А. Ю. Ольшанским и его учениками с использованием этого языка. В частности, можно упомянуть работу В. С. Губы [24], в которой с помощью техники А. Ю. Ольшанского впервые были построены конечно порожденные полные группы, т.е. группы, в которых из каждого элемента можно извлечь корень любой степени $n$.

Здесь следует также отметить очень важные результаты, которые были получены в 90-е годы независимо С. В. Ивановым [35] 
и И. Г. Лысенком [25]. Они установили бесконечность свободных периодических групп $B(m, n)$ для достаточно больших четных периодов $n$.

При этом им пришлось попутно исследовать структуру конечных подгрупп группы $B(m, n)$ и добавить соответствующие свойства к рассматриваемой системе утверждений, доказываемых совместной индукцией. В процессе доказательства выяснилось, что для применения метода в четном случае нужно потребовать, чтобы период $n$ имел достаточно большой четный множитель: у Лысенка - не менее $2^{4}$, а у Иванова - не менее $2^{9}$. Кстати, внимательный читатель легко заметит, что, анонсируя свой результат, С. В. Иванов в 1992 году еще не знал, что такое условие надо будет наложить на период. Иначе он не стал бы акцентировать внимание на случае, когда этот множитель равен 2 или 4. Позже И. Г. Лысенок показал, что именно в этих случаях метод не удается применить. Впрочем, в своей итоговой статье Иванов это дело исправил.

Любопытно отметить, что в результате статья Лысенка с доказательством бесконечности групп $B(m, n)$ при $n=2^{4} k \geqslant 8000$ (в английской версии) заняла 202 страницы, а статья Иванова [35] с доказательством для периодов $n=2^{9} k \geqslant 2^{4} \cdot 8$ заняла 307 страниц. Все дело в том, что оба автора использовали более экономный язык диаграмм Ван Кампена, а схемы индукции они использовали разные: Иванов проводил индукцию по схеме, изложенной в книге Ольшанского [26], а Лысенок - по схеме, изложенной в книге [10].

\section{Список литературы}

[1] Burnside W., "On an unsettled question in the theory of discontinuous groups" // Quart. J. Pure Appl. Math., 1902, 33, 230-238.

[2] Burnside W., "On criteria for the finiteness of the order of linear substitutions" // Proc. London Math. Soc., 1905, 3, 435-440.

[3] Новиков П.С., "О периодических группах" // Докл. АН CCCP, 1959, 127(4), 749-752. 
[4] Новиков П. С., Адян С.И., "О бесконечных периодических группах. I, II, III" // Изв. АН СССР. Сер. матем., 1968, 32(1), 212-244; 32(2), 251-524; 32(3), 709-731.

[5] Новиков П.С., Адян С. И., "Определяющие соотношения и проблема тождества для свободных периодических групп нечетного порядка" // Изв. АН СССР. Сер. матем., 1968, 32(4), 971-979.

[6] Новиков П. С., Адян С. И., "О коммутативных подгруппах и проблеме сопряженности тождества для свободных периодических групп нечетного порядка" // Изв. АН СССР. Сер. матем., 1968, 32(5), 1176-1190.

[7] Адян С. И., "Бесконечные неприводимые системы групповых тождеств" // Докл. АН СССР, 1970, 190(3), 499-501; Изв. АН СССР. Сер. матем., 1970, 34(4), 719-734.

[8] Адян С. И., "О некоторых группах без кручения" // Изв. АН СССР. Сер. матем., 1971, 35(3), 459-468.

[9] Адян С. И., "О подгруппах свободных периодических групп нечетного показателя" // Трудъ МИАН, 1971, 112, 64-72.

[10] Адян С.И., Проблема Бернсайда и тождества в группах. М.: Наука, 1975.

[11] Адян С. И., "Периодические произведения групп" // Трудъ МИАН, 1976, 142, 3-21.

[12] Адян С. И., "О простоте периодических произведений групп" // Докл. АН СССР, 1978, 241(1), 745-748.

[13] Адян С.И., "Нормальные подгруппы свободных периодических групп" // Изв. АН СССР. Сер. матем., 1981, 45(5), 1139-1149.

[14] Адян С. И., "Аксиоматический метод построения групп с заданными свойствами" // УМH, 1977, 32(1), 3-15.

[15] Адян С.И., // Случайные блуждания на свободных периодических группах, // Изв. АН СССР. Сер. матем., 1982, 46(6), 1139-1149. 
[16] Адян С. И., "Исследования по проблеме Бернсайда и связанным с ней вопросам" // Трудъ МИАН, 1984, 168, 171-196.

[17] Адян С. И., Лысенок И. Г., "О группах, все собственные подгруппы которых конечные циклические" // Изв. АН СССР. Сер. матем., 1991, 55(5), 933-990.

[18] Алешин С.В., "Конечные автоматы и проблема Бернсайда о периодических группах" // Матем. заметки, 1972, 11(3), 319-328.

[19] Атабекян В. С., Иванов С. В., Два замечания о группах ограниченного периода. Деп. в ВИНИТИ 30.03.1987, № 2243-В87.

[20] Голод Е. С., "О ниль-алгебрах в финитно-аппроксимируемых группах" // Изв. АН СССР. Сер. матем., 1964, 28(2), 273276.

[21] Григорчук Р.И., "О проблеме Бернсайда о периодических группах" // Функи. анализ и его прилож., 1980, 14(1), 53-54.

[22] Григорчук Р. И., “Симметрические случайные блуждания на дискретных группах" // Многокомпонентные случайные системы. - М.: Наука, 1978, 132-152.

[23] Губа В. С., "Конечно порожденная полная группа" // Изв. АН СССР. Сер. матем., 1986, 50(5), 50-67.

[24] Губа В. С., Построение групп с новыми свойствами с помощъю диаграмм сокращения. Автореферат канд. дисс. - М.: Изд-во МГУ, 1989.

[25] Лысенок И.Г., "Бесконечные бернсайдовы группы четного периода" // Изв. РАН. Сер. матем., 1996, 60(3), 3-224.

[26] Ольшанский А. Ю., Геометрия определяющих соотношений в группах. - М.: Наука, 1989.

[27] Санов И.Н., "Решение проблемы Бернсайда для показателя 4" // Ученые записки ЛГУ. Сер. матем., 1940, 10, 166170.

[28] Санов И.Н., "Установление связи между периодическими группами с периодом числом и кольцами Ли" // Изв. $A H$ СССР. Сер. матем., 1952, 16(1), 23-58. 
[29] Чандлер Б., Магнус В., Развитие комбинаторной теории гpynn. - М.: Мир, 1985.

[30] Adian S. I., "On the word probem for groups defined by periodic relations" // Lecture Notes in Math., 1980, 806, 41-46.

[31] Adyan S. I., Lysionok I. G., "The method of classification of periodic words and the Burnside problem" // Contemp. Math., 1992, 131 (Part 1), 13-28.

[32] Britton J. L., "The existence of infinite Burnside groups" // Stud. Logic Found. Math., 1973, 71, 67-348.

[33] Britton J.L., "Erratum: The existence of infinite Burnside groups" // Stud. Logic Found. Math., 1980, 95, 71.

[34] Hall M. jun., "Solution of the Burnside problem for exponent six" // Illinois J. Math., 1958, 2, 764-786.

[35] Ivanov S. V., "The free Burnside groups of sufficiently large exponents" // Int. J. Algebra Comput., 1994, 4, 1-307.

[36] Kesten H., "Symmetric random walks on groups" // Trans. Amer. Math. Soc., 1959, 92, 336-354.

[37] Magnus W., "A connection between the Baker-Hausdorf formula and a problem of Burnside" // Ann. of Math. (2), 1950, 52, 111$126 ; 1953, \mathbf{5 7}, 606$.

[38] Shur I., "Über Gruppen periodisher Linearer Substitutionen" // S. Ber. Preuss. Akad., 1911, 619-627. 